\title{
Combination therapies in the management of type 2 diabetes: the use of insulin degludec/liraglutide
}

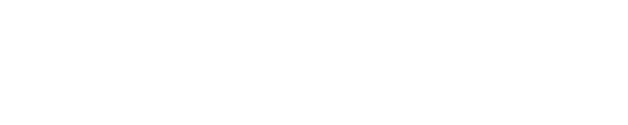

\section{Molly G Minze' \\ Lisa M Chastain ${ }^{2}$}

'School of Pharmacy, Texas Tech University Health Sciences Center, Abilene, ${ }^{2}$ Department of Pharmacy Practice, School of Pharmacy, Texas Tech University Health Sciences Center, Dallas, TX, USA
Correspondence: Molly G Minze School of Pharmacy, Texas Tech University Health Sciences Center, I7I8 Pine Street, Abilene, TX 7960I, USA

Tel +l 3256960453

Fax + I 3256763824

Email molly.minze@ttuhsc.edu

\begin{abstract}
The global burden of type 2 diabetes is estimated to currently affect over 350 million people worldwide and is anticipated to continue increasing over the next 20 years. Current treatment guidelines recommend the choice of pharmacotherapy based upon patient-specific parameters, with combination therapy for patients with a hemoglobin Alc level $\geq 9 \%$. A new combination therapy of insulin degludec + liraglutide provides a long-acting basal insulin with a glucagon-like peptide agonist. In clinical trials, this combination product has reduced hemoglobin A1c and fasting plasma glucose more than the individual agents alone. Further advantages observed with this combination include weight loss and decrease in hypoglycemia compared to basal insulin alone.
\end{abstract}

Keywords: insulin degludec, liraglutide, type 2 diabetes, basal insulin, glucagon-like peptide agonist, injectable therapies

\section{Introduction}

The global burden of type 2 diabetes is estimated to currently affect approximately 387 million people worldwide with a prevalence of $8.3 \%$ of the worldwide population. ${ }^{1}$ This rate is expected to increase by an additional 205 million people worldwide by 2035 . Individualized patient treatment approaches are endorsed in the most recent recommendations from the American Diabetes Association, American Association of Clinical Endocrinologists, and the UK National Institute for Healthcare and Excellence. The choice of therapy is contingent on patient-specific attributes and needs, which encourages providers to use oral pharmacotherapies such as metformin, sulfonylureas, thiazolidinediones, dipeptidyl peptidase-4 inhibitors, or injectable therapies, including glucagon-like peptides (GLP-1) or insulin therapy. ${ }^{2-4}$ Despite concerted efforts and guidance, up to $49 \%$ of patients do not meet their hemoglobin A1c (HbA1c), blood pressure, or lipid goal..$^{5}$ Initial combination therapy is recommended for patients with higher HbA1c levels at baseline (ie, $\geq 9 \%$ ) who are unlikely to gain control with monotherapy. ${ }^{2}$ Combination therapy using basal insulin, in addition to a GLP-1 agonist, has been effective and equal to basal and prandial insulin. ${ }^{6-8}$

A search was performed within PubMed using the key terms "insulin degludec" and "liraglutide". This review explores the combination use of insulin degludec in fixed combination with liraglutide.

\section{Pharmacology Basal insulin and GLP-I agonists}

Basal insulin provides sustained glucose control through its long duration of action and steady exogenous insulin delivery without a peak. ${ }^{9}$ Glucagon-like protein-1 agonists are 
designed to mimic the incretin hormone GLP-1 and gastric inhibitor polypeptide, both of which are produced after eating a meal. ${ }^{10}$ These incretin hormones promote insulin secretion and suppress the production of glucagon through a glucosedependent mechanism. ${ }^{10}$ Basal insulin reduces fasting and post absorptive blood glucose, whereas GLP-1 agonists decrease postprandial glucose via gastric emptying inhibition, stimulation of glucose-dependent insulin secretion, and suppression of glucagon production. ${ }^{6}$

Insulin degludec, a new basal insulin analog administered subcutaneously once daily, provides a constant and steady insulin exposure with less within-patient variability compared with insulin detemir and insulin glargine. ${ }^{10-13}$ Insulin degludec has been designed as an ultra-long-acting basal insulin that improves the following limitations of other marketed basal insulins: short half-life compared to duration of action, restriction of patient lifestyle secondary to need for consistent timing of injection from day-to-day, and improved simulation of physiologic distribution of endogenous insulin. ${ }^{13}$ After subcutaneous injection, insulin degludec forms a soluble depot with slow and continuous absorption secondary to dihexamer self-association into multihexamer chains. ${ }^{12,13}$ Zinc gradually diffuses from the multihexamers allowing a slow and steady delivery into circulation. ${ }^{13}$ Insulin degludec is also highly protein bound ( $\left.>99 \%\right)$ but its concentration is very low in circulation compared to albumin ( $>10,000$ fold), occupying $<0.01 \%$ of albumin molecules. ${ }^{12}$ Because of this, insulin degludec will not be influenced by other albumin-bound drugs or large changes in albumin concentrations. ${ }^{12}$ Insulin degludec has a half-life of $\sim 25$ hours after subcutaneous administration, a duration of action exceeding 42 hours, and attainment of a steady state within 3 days. ${ }^{12}$ Once at a steady state, the glucose-lowering effect of insulin degludec is less variable than other basal insulins secondary to overlap of daily injections. ${ }^{10}$ Dose titrations may be initiated once weekly. ${ }^{12}$ In special populations such as the elderly, renally impaired, and hepatically impaired, insulin degludec's pharmacokinetic and pharmacodynamic properties are preserved. ${ }^{12}$ Adverse effects observed with insulin degludec are consistent with other insulin products and include hypoglycemia, and weight gain. ${ }^{14-17}$ Additionally, a case report using insulin degludec successfully in an otherwise insulin allergic patient has been reported in the literature. ${ }^{18}$

Liraglutide is a subcutaneously injected GLP-1 analog that contains one amino acid substitution compared to endogenous GLP-1, thereby protecting liraglutide against degradation by dipeptidyl peptidase-4 enzymes and neutral endopeptidases in vivo. ${ }^{19,20}$ Because of this substitution, liraglutide has a oncedaily dosing schedule and an extended half-life of $\sim 13$ hours compared to endogenous GLP-1's half-life of 1.5-2 minutes. ${ }^{19}$ Upon binding to the GLP-1 receptor, liraglutide causes dosedependent insulin release through stimulation of pancreatic $\beta$-cells in patients with elevated glucose levels. With this, postprandial glucose levels are reduced. Liraglutide does not stimulate pancreatic $\alpha$-cells, and therefore decreases inappropriately high glucagon secretion in a glucose-dependent manner, blocking hepatic glucose output from glucagon. Moreover, liraglutide delays gastric emptying and reduces hunger, thereby decreasing body weight and body fat mass. Over a 24-hour dosing period, liraglutide provides sustained fasting and lowering of postprandial glucose. Patients may develop anti-liraglutide antibodies, but these antibodies do not appear to reduce efficacy or impact safety. ${ }^{19}$ After subcutaneous administration, the maximum plasma concentration is achieved between 8 and 12 hours. ${ }^{19}$ The mean volume of distribution after subcutaneous administration is $11-17 \mathrm{~L}$, with an approximate $55 \%$ bioavailability. Liraglutide is metabolized to large proteins, without a major organ system route of elimination. Pharmacokinetics of liraglutide are not influenced by age, race, sex, ethnicity, or body mass index (BMI). In patients with mild-to-moderate hepatic impairment, liraglutide exposure was slightly lower than in healthy volunteers, but significantly decreased in patients with severe hepatic impairment defined as a Child-Pugh score $>9$. In patients with renal impairment, liraglutide exposure was reduced compared to healthy volunteers, although no specific dosage reduction was recommended as per the manufacturer. ${ }^{19,21}$ A low potential for drug-drug interactions exists with liraglutide secondary to its cytochrome P450 neutrality and plasma protein binding. ${ }^{19,20}$ Additionally, no clinically relevant drug interactions have been observed secondary to the delay in gastric emptying. Adverse effects that occur with liraglutide use include nausea, vomiting, and diarrhea. ${ }^{22}$

\section{Degludec and liraglutide combination}

When basal insulin and a GLP-1 agonist are used in combination, they target complementary blood glucoselowering mechanisms. ${ }^{6}$ Degludec and liraglutide have recently been formulated in combination together as a once-daily single injection prefilled pen with degludec 100 units $/ \mathrm{mL}+$ liraglutide $3.6 \mathrm{mg} / \mathrm{mL}$ for use in type 2 diabetes. ${ }^{23,24}$ Degludec + liraglutide (IDegLira) is approved in the European Union and has filed for approval in the USA. ${ }^{23}$ In a pharmacokinetic study, IDegLira had preserved kinetic properties compared to monotherapy of the individual agents. ${ }^{25}$ Also, the pharmacodynamics properties of the combination product have remained the same as those of the 
individual products. ${ }^{24}$ Like the individual products, the most common adverse effects seen with the combination agent are hypoglycemia and gastrointestinal symptoms, such as nausea, diarrhea, vomiting, and constipation. ${ }^{24}$

\section{Clinical studies Efficacy}

Several clinical trials have evaluated the efficacy of the combination of insulin degludec + liraglutide for the treatment of diabetes in differing background antidiabetic regimens (insulin naïve, patients previously treated with metformin, or patients previously on basal insulin).

DUAL-I was a treat-to-target, open-label, noninferiority trial evaluating efficacy (change in HbA1c after 26 weeks) of IDegLira compared to its components in insulin-naïve patients. ${ }^{26}$ Subjects with type 2 diabetes, HbA1c 7\%-10\%, BMI $\leq 40 \mathrm{~kg} / \mathrm{m}^{2}$ treated with metformin, with or without pioglitazone were randomized to once-daily IDegLira $(\mathrm{n}=834)$, insulin degludec $(\mathrm{n}=414)$, or liraglutide $1.8 \mathrm{mg} /$ day $(n=415)$. IDegLira and degludec were titrated twice weekly to fasting plasma glucose (FPG) of $72-90 \mathrm{mg} / \mathrm{dL}$; however, IDegLira was capped at a maximum of 50 dose steps (50 units IDeg $+1.8 \mathrm{mg}$ liraglutide). IDegLira produced a significantly greater $\mathrm{HbA} 1 \mathrm{c}$ reduction from baseline $(-1.9 \%)$ compared with IDeg $(-1.4 \%$ from baseline) or liraglutide $(-1.3 \%)$. IDegLira was noninferior to insulin degludec (estimated treatment difference [ETD], $-0.47 \%, 95 \%$ confidence interval [CI] $[-0.58$ to -0.36$], P<0.0001)$ and superior to liraglutide (ETD $-0.64 \%, 95 \%$ CI $[-0.75$ to -0.53$]$, $P<0.0001)$. DUAL-I also saw greater numbers of patients achieving $\mathrm{HbA} 1 \mathrm{c}<7 \%$ in the IDegLira group after 26 weeks compared to IDeg $(81 \%$ vs $65 \%, P<0.0001)$ or liraglutide $(60 \%, P<0.001)$. Another benefit seen in this trial was a weight loss of $0.5 \mathrm{~kg}$ in the patients in the IDegLira group compared with a weight increase of $1.6 \mathrm{~kg}$ in patients in the IDeg group $(P<0.0001)$. Lastly, at 26 weeks, mean insulin dose was $28 \%$ lower with IDegLira than with IDeg (38 vs 53 units, $P<0.0001){ }^{26}$

DUAL-I trial authors also published data from the extension trial, which followed subjects for an additional 26 weeks to assess the sustainability of the treatment response of IDegLira over 52 weeks. ${ }^{27}$ The 52-week secondary endpoints included change from baseline in $\mathrm{HbA} 1 \mathrm{c}$, percentage of patients reaching $\mathrm{HbA} 1 \mathrm{c}<7 \%$ and $\leq 6.5 \%$ as well as changes from baseline in FPG, body weight, insulin dose, and 9 point self monitoring blood glucose (SMBG) profiles. There were 1,311 patients who continued into the DUAL-I extension phase (665 IDegLira/333 IDeg/313 Lira), which was approximately $78 \%$ of the population from the original trial. At the end of the 52 weeks, mean HbA1c concentration remained significantly lower for the IDegLira group compared to the IDeg group $(-1.84 \%$ vs $-1.40 \%, P<0.0001)$ and liraglutide $(-1.21 \%, P<0.0001)$. Additionally, a greater number of subjects in the extension trial achieved maintained $\mathrm{HbA} 1 \mathrm{c}<7 \%$ if receiving IDegLira (78.2\%) compared to IDeg $(62.5 \%)$ or liraglutide alone $(56.5 \%)$. Also noted in the extension trial was that body weight remained relatively stable throughout the extension with IDegLira $(-0.4 \mathrm{~kg})$ and liraglutide $(-3.0 \mathrm{~kg})$, but increased by a total of $2.3 \mathrm{~kg}$ from baseline in the IDeg group. Mean daily insulin doses of the insulin degludec and liraglutide components were significantly lower in the IDegLira ( $39 \pm 13$ units $/ 1.4 \pm 0.5 \mathrm{mg}$ ) group when compared with either product alone (62 \pm 42 units insulin degludec, $1.8 \pm 0.7 \mathrm{mg}$ liraglutide). ${ }^{27}$

The DUAL-II trial set out to determine the contribution of the liraglutide component of IDegLira in a 26-week open-label study comparing diabetics (HbA1c 7.5\%-10.0\%, BMI $\geq 27 \mathrm{~kg} / \mathrm{m}^{2}$, uncontrolled on 20-40 units of basal insulin + metformin \pm secretagogues) randomized to IDegLira + metformin $(n=199)$ or IDeg + metformin $(n=199) .{ }^{28}$ At randomization, participants were allowed to remain on metformin while basal insulin was switched to either IDeg 16 units or IDegLira 16 dose steps (16 units degludec $+0.6 \mathrm{mg}$ liraglutide). Doses were adjusted biweekly to a target of FPG 72-90 mg/dL and were capped at either 50 units or 50 dose steps to evaluate the impact of the liraglutide component to glycemic control. At study end, IDegLira achieved a $1.9 \% \mathrm{HbA} 1 \mathrm{c}$ reduction compared to $0.9 \%$ reduction in IDeg group (ETD 1.1\%, 95\% CI [-1.3 to -0.8$]$, $P<0.0001)$ despite equivalent IDeg doses ( $\sim 5$ units). Patients in the IDegLira group had a greater chance of achieving HbA1c < 7\% (60\% IDegLira vs 23\% IDeg, with a higher estimated odds of achieving goal, $P<0.0001)$ as well as the percentage of patients to achieve this goal without hypoglycemia or weight gain (40\% IDegLira vs $8.5 \%$ IDeg, $P<0.0001)$. FPG and 9 point SMBGs were also significantly lower in IDegLira group. Lastly, $2.7 \mathrm{~kg}$ weight reduction was seen in the IDegLira group vs no change in the IDeg group $(P<0.001){ }^{28}$

BEGIN: VICTOZA ADD-ON was an open-label, treatto-target study investigating the efficacy and safety of adding liraglutide once-daily vs insulin aspart once-daily at largest meal to insulin degludec + metformin. ${ }^{29}$ Study subjects were randomized to IDeg + Lira $(n=88)$ or IDeg + IAsp $(n=89)$ for 26 weeks, targeting FPG 71-89 mg/dL. The primary endpoint in this trial was a change in $\mathrm{HbA} 1 \mathrm{c}$ after 26 weeks 
with secondary endpoints, including change in FPG, body weight, and 9 point SMBG profile. At study end, patients in the IDeg + Lira achieved HbAlc reduction of $0.76 \%$ points compared to $0.39 \%$ point reduction in IDeg + IAsp (ETD $-0.32 \%$ points, $95 \%$ CI $[-0.53$ to -0.12$], P=0.0024)$. Between groups, there was no statistically significant difference in IDeg + Lira vs IDeg + IAsp achieving HbA1c $<7 \%$ ( $58 \%$ vs $44.9 \%$, respectively); however, there were significantly more patients in the IDeg + Lira group able to achieve HbA1c $<7 \%$ without hypoglycemia (estimated odds ratio [OR] 5.57, 95\% CI [2.67-11.63], $P<0.001)$ and without hypoglycemia and weight gain (estimated OR 13.79, 95\% CI [5.24-36.28], $P<0.0001)$. No differences were seen between groups with respect to FPG and 9 point SMBG profiles; however, significant weight difference seen at 26 weeks with the IDeg + Lira group losing $2.8 \mathrm{~kg}$ compared to a $0.9 \mathrm{~kg}$ gain in body weight in the IDeg + IAsp group $(P<0.0001)$. Although this study did not evaluate the combination product of insulin degludec, it did provide insight into potential benefits of adding a long-acting GLP-1 agonist to basal insulin compared to once-daily bolus insulin added to basal insulin. ${ }^{29}$

Freemantle et $\mathrm{al}^{30}$ describe a pooled analysis of the five completed clinical trials from the manufacturer of IDegLira. Using patients from the DUAL-II trial and four other Novo Nordisk sponsored trials, an estimate of indirect treatment effects was obtained with the primary outcome of change in $\mathrm{HbAlc}$ from baseline to study end. Secondary outcomes included hypoglycemia, change in baseline body weight and BMI, and percentage of patients achieving $\mathrm{HbA} 1 \mathrm{c}<7 \%$ with or without hypoglycemia or weight gain. The analysis compared basal insulin products with IDegLira in patients with type 2 diabetes not controlled by basal insulin from the Novo Nordisk clinical trial database. The treatment outlined in four of the trials included insulin titration to a FPG target of $72-90 \mathrm{mg} / \mathrm{dL}$ (4-5 mmol/L), whereas the remaining trial reduced pretrial basal insulin doses by $20 \%$, which is similar to current practice trends when liraglutide is added to basal insulin. A significant lowering of estimated HbAlc occurred in the IDegLira group $(-1.68 \%, 95 \%$ CI $[-1.82$ to -1.54$])$ than in the other groups, including: GLP-1+ basal insulin, basal-bolus insulin therapy, and uptitrated insulin glargine groups $(-1.33 \%, 95 \%$ CI $[-1.48$ to -1.18$])$, basal-bolus insulin therapy $(-1.39 \%, 95 \%$ CI $[-1.64$ to -1.13$])$, and uptitrated insulin glargine group $(-1.03,95 \%$ CI $[-1.14$ to -0.93$]$ ). Body weight and BMI also showed significant reductions in the IDegLira group (body weight $-2.88 \mathrm{~kg}$,
$95 \%$ CI [ -3.39 to -2.27$],$ BMI $-1.02 \mathrm{~kg} / \mathrm{m}^{2}, 95 \%$ CI $[-1.19$ to -0.84$]$ ) compared to all other groups. ${ }^{30}$

\section{Safety and tolerability data}

The trials (DUAL-I, DUAL-I extension, DUAL-II, and BEGIN: VICTOZA ADD ON) also looked at the safety and tolerability of the combination of insulin degludec and liraglutide compared to the individual components. Tolerability outcomes generally included hypoglycemic episodes and gastrointestinal symptoms, while safety outcomes included pancreatitis and increased concentrations of amylase and lipase, or incidence of thyroid carcinoma. In DUAL-I, confirmed hypoglycemia was significantly greater in the IDegLira group when compared to the liraglutide group (Estimated rate ratio [ERR] 7.61, 95\% CI [5.17-11.21], $P<0.0001$ ) but less frequent than the IDeg group (ERR 0.68, 95\% CI [0.53-0.87], $P=0.0023) .{ }^{26}$ Similar results were seen in the DUAL-I extension trial with lower rates of confirmed hypoglycemia per 100-patient years of exposure (PYE) in the IDegLira group when compared to IDeg (Rate ratio [RR] $0.63, P<0.0001)$ but greater rates of hypoglycemia when compared to the liraglutide group (RR 8.52, $P<0.0001$ ). Nocturnal hypoglycemia was greater in the IDegLira group in the extension study when compared to liraglutide (RR 11.99, $P<0.0001)$ but no significant differences were noted when compared to IDeg. ${ }^{27}$ DUAL-II study found a numerically greater incidence of confirmed hypoglycemia with the IDegLira compared to IDeg alone, however, these results were not statistically significant (RR $0.66, P=0.13$ ). Additionally, nocturnal hypoglycemia rates were low and similar between groups. ${ }^{28}$ Hypoglycemia results remain consistent, even in the BEGIN: VICTOZA ADD-ON trial, with a rate of confirmed hypoglycemia of 1.00 episode per PYE with IDegLira compared to 8.15 episodes per PYE in IDeg + IAsp (ERR 0.13, 95\% CI [0.08-0.21], $P<0.0001)$ and nocturnal hypoglycemia identified at 0.17 per PYE in IDegLira compared to 1.11 per PYE with IDegAsp (ERR 0.14, 95\% CI [0.05-0.40], $P=0.0002) .{ }^{29}$ These results of hypoglycemic safety suggest that while the combination of IDeg + liraglutide might carry a higher risk of hypoglycemia compared to liraglutide or IDeg alone or IDeg plus bolus insulin, the risk of hypoglycemia is reduced, suggesting mitigation of the hypoglycemic effects even when targeting fairly strict glycemic goals.

Aside from hypoglycemia there were additional adverse effects seen in these trials, associated with the drugs, specifically liraglutide, that might deter adherence for patients. In general, IDegLira has been well tolerated, with adverse events (AEs) categorized as mild or moderate in 
severity. The most frequently reported adverse effects were generally headache ( $\sim 10 \%$ in most trials), nasopharyngitis ( $9 \%$ in most trials), or gastrointestinal disorders. ${ }^{26-29} \mathrm{In}$ DUAL-I, nausea occurred with greater frequency in the liraglutide group (20\%) when compared to the IDeg (4\%) or IDegLira $(9 \%)$ groups. The reduced level of nausea observed in the IDegLira group was likely related to the overall slower dose escalation and lower ending dose. Per study investigators, a higher proportion of subjects withdrew from the liraglutide group for gastrointestinal (GI) AEs when compared to the IDegLira or IDeg groups. ${ }^{26}$ In DUAL-I extension, AE reporting remained similar to the first 26 weeks, with overall lower rates of AEs reported with IDegLira or IDeg compared to liraglutide (407.9 vs 383.3 vs 507.3 per 100 PYE, respectively) and specifically the incidence of nausea in the extension study remained consistent with the first 26 weeks. ${ }^{27}$ DUAL-II found similar results when comparing IDegLira to IDeg, with nausea occurring in $6.5 \%$ and $3.5 \%$ of subjects, respectively, and was more frequent in the first 12 weeks. However, study authors indicated that there were no drug withdrawals due to GI side effects in this 26 -week trial. ${ }^{28}$ Similarly, when comparing GI symptoms in the BEGIN: VICTOZA ADD ON trial, nausea was more frequent in the IDeg + Lira group at two distinct time points, one at baseline and another when liraglutide was titrated, but symptoms declined within 2 weeks. At study endpoint, only 3\% of subjects in the IDeg + Lira reported nausea and there were no study drug discontinuations related to nausea. ${ }^{29}$

In the Freemantle pooled analysis, overall rates of hypoglycemia and nonsevere hypoglycemia appeared significantly lower with IDegLira (0.99, 95\% CI [0.63-1.54], $P=0.95)$ compared to IDegLira vs basal-bolus $(0.12,95 \% \mathrm{CI}$ [0.07-0.2], $P<0.0001)$ and IDegLira vs uptitrated glargine $(0.43,95 \%$ CI [0.3-0.62], $P<0.0001)$ with severe hypoglycemia occurring too infrequently to show a meaningful statistical difference. ${ }^{30}$

In addition to known and documented adverse effects, there are also noted safety concerns with the individual agents in this drug combination. The class of GLP-1 agonists is known to carry a risk of pancreatitis and medullary thyroid carcinomas, thus each of these trials were monitored for such incidence. With respect to the issue of pancreatitis or elevated amylase and or lipase levels, the results were somewhat mixed between these trials. In DUAL-I, 18 cases of pancreatitis occurred; however, only one of these was confirmed to be acute pancreatitis but was judged as unlikely related to study drug. ${ }^{26}$ In the DUAL-I EXTENSION, five cases of pancreatitis, two with IDegLira (both treatment-emergent), two cases with liraglutide (one treatment-emergent), and one nontreatment-emergent case with IDeg were identified. Of these cases, only one was positively adjudicated in the liraglutide group and one in the insulin degludec group. This trial also followed trends of amylase and lipase levels in subjects over 52 weeks and found 16 events of increased lipase and/or amylase with only one adjudicated as increased pancreatitis in the liraglutide group..$^{27}$ There were no thyroid medullary carcinomas reported in either the DUAL-I or extension trial. ${ }^{26,27}$ Similarly, DUAL-II did not find any confirmation of thyroid carcinoma or pancreatitis, but found greater changes in amylase and lipase in the IDegLira group (seven vs two patients in IDeg), though no occurrence was associated with pancreatitis. ${ }^{28}$ In BEGIN-VICTOZA ADD ON only one case of pancreatitis occurred in the IDeg + IAsp arm (deemed unlikely to be related to either study drug) and no cases in the IDeg + Lira group. ${ }^{29}$

Based on the data presented earlier, it appears that the addition of liraglutide to basal insulin degludec provides many potential benefits of diminishing some of the main side effects associated with basal insulin alone, such as weight loss and hypoglycemia. Additionally, the presence of the basal insulin allows for lower doses of GLP-1 agonist to reduce the impact of gastrointestinal side effects. Not only is this new option effective at glycemic reduction, but it also seems to be a safe option with low incidence of pancreatitis truly attributable to the combination. However, individual patient factors must be considered on a case by case basis, as the combination of insulin degludec + liraglutide might not be the optimal choice for all patients. When applying the clinical trial data, an ideal patient to receive insulin degludec + liraglutide would be one with disease progression who is no longer controlled with oral medications and basal insulin alone. It would seem prudent to consider the use of a GLP-1 agonist combined with basal insulin therapy when considering whether to add meal-time bolus insulin as the use of a GLP-1 analog would provide postprandial glycemic control to all meals, compared to stepwise addition of bolus insulin, resulting in three separate injections for meals in addition to the basal insulin. In fact, many widely recognized diabetes treatment algorithms, such as American Diabetes Association and the American Academy of Clinical Endocrinology, now indicate the combination of basal insulin + GLP1 agonist be considered at this step..$^{31,32}$ With the combination product of IDegLira, the patient is able to receive both prandial and postprandial glucose reduction with only one injection. 


\section{Patient perspectives}

Patients living with diabetes experience burdens and challenges from the disease that often negatively impact their health-related quality of life (QoL), such as higher rates of morbidity and mortality. ${ }^{33}$ Exogenous insulin does improve glucose control and can improve prognosis, but does not eliminate physical or psychosocial concerns of the disease. Common justifications that decrease QoL include fear of hypoglycemia, fear of self-injection, anxiety regarding inflexible or complex dosing regimens, and embarrassment of diagnosis. At this time, patient-centered perspectives with the use of degludec + liraglutide are not available. However, perspectives are available with the individual agents degludec and liraglutide, as presented in the paragraphs below.

In a meta-analysis of Phase III trials, health-related QoL scores were evaluated in patients with diabetes who received insulin degludec vs insulin glargine. ${ }^{34}$ Six trials included in the meta-analysis were Phase IIIa clinical trials with IDeg once-daily vs IGlar once-daily that included QoL analysis, and were randomized, controlled, open-label trials of 26 or 52 weeks that included a total of 4,001 patients. At baseline and end-of-trial, patients completed a Short Form 36 (SF-36) version-2 health questionnaire, where an increase in SF-36 score indicated improvement in health, but are not based on individual preference. The SF-36 scores were then converted to EuroQol-5D health utility scores, which can be interpreted in a range of -0.59 (worst health) to 1 (best health). The average age of the patient in the combined population within the six trials assessed was $56 \pm 11.7$ years, with a duration of diabetes of $12.1 \pm 8.4$ years, with $\mathrm{HbA} 1 \mathrm{c}$ of $8.2 \pm 0.9 \mathrm{mmol} / \mathrm{mol}$, FPG $166 \pm 54.1 \mathrm{mg} / \mathrm{dL}$, and BMI of $30.0 \pm 5.3 \mathrm{~kg} / \mathrm{m}^{2}$. Significant improvement in EuroQol-5D scores was seen in the IDeg treatment groups of 0.005, (95\% CI [0.0006-0.009]) compared to IGlar $(P<0.024)$. These results show a statistically significant improvement in health utility with IDeg compared to IGlar, although the authors admit it is a modest improvement.

In patients with type 1 diabetes, health status was assessed through SF-36 during a randomized, 16-week open-label trial. ${ }^{35}$ Patients with type 1 diabetes with an average age of 45.8 years, $\mathrm{HbA1c} 8.4 \%$, and BMI $26.9 \mathrm{~kg} / \mathrm{m}^{2}$ were randomized to receive glargine $100 \mathrm{U} / \mathrm{mL}(\mathrm{n}=59)$ or degludec $100 \mathrm{U} / \mathrm{mL}(\mathrm{n}=59)$, along with mealtime insulin aspart. The mental score improved significantly in the degludec group after 16 weeks as compared to the glargine group by 3.01 (95\% CI 0.32-5.7). Contributing to this increase was social functioning by 8.04 (significant but moderate difference, $95 \%$ CI 1.89-14.18), and an increase in the mental health domain by 2.46 (significant but small-to-medium difference, $95 \%$ CI 0.1-4.82). The physical component score did not show statistical significance between groups with +0.66 difference (95\% CI -2.3 to -3.62 ). Insulin degludec improved SF-36 mental health scores in type 1 patients receiving insulin degludec compared to those receiving insulin glargine.

Patient medication adherence has not been researched with the combination of insulin degludec and liraglutide; however, adherence to each component individually has been investigated. A single-center study evaluated adherence to insulin therapy through analyzing treatment persistence and daily adherence in patients with type 2 diabetes who were insulin naïve. ${ }^{36} \mathrm{~A}$ total of 433 patients with an average age of 55.5 \pm 13 years received basal, basal-bolus, or premixed insulin via pen device. Patients were phone-interviewed 6 months postinitiation of insulin. Insulin nonadherence occurred in $44.3 \%$ ( $n=192)$ of patients with an all-cause treatment discontinuation of $24 \%(\mathrm{n}=104)$, and daily insulin nonadherence of $20.3 \%$ ( $n=88)$. Eighty-seven patients (20.1\%) withdrew from treatment, with $75.9 \%$ of withdrawals were at the patient's discretion. Patient nonpersistence occurred in $13.7 \%$ of patients and was based on a physician's decision. In patients who were persistent to insulin therapy, daily insulin adherence resulted in lower daily insulin doses $(P=0.03)$ and self-measured postprandial blood glucose readings $(P=0.04)$. The premixed and basal insulin groups had improved treatment adherence compared with basal-bolus regimens $(P=0.04)$.

Patient adherence and persistence to liraglutide oncedaily in adult (age $\geq 18$ years) type 2 diabetes $(n=1,321)$ was studied in a retrospective cohort claims data analysis. ${ }^{37}$ Adherence was defined as proportion of days covered with a score of $\geq 0.8$ deemed nonadherent, and nonpersistence was defined by a $>90$-day gap in therapy. The average proportion of days covered was 0.59 with patient adherence of $34 \%$ and over 12 months, $60 \%$ of patients were persistent. Characteristics of a patient achieving HbAlc goals were those who were adherent and persistent compared to those who were nonadherent and nonpersistent (HbA1c $8.08 \%$ vs $8.29 \%, P=0.033$ ).

\section{Conclusion}

The combination of insulin degludec + liraglutide combines a new basal insulin analog with a GLP-1 agonist. Clinical trials provide data supporting improved glycemic targets (HbA1c, FPG) with this combination therapy more than with the individual products alone. Further favorable effects shown include weight loss, lower basal insulin dose, and with 
less hypoglycemia than basal insulin alone. Safety concerns of pancreatitis and medullary thyroid carcinomas associated with the combination insulin degludec + liraglutide showed few cases of pancreatitis and no cases of medullary thyroid carcinomas within the clinical trials completed to date. Overall, the combination of insulin degludec + liraglutide provides beneficial $\mathrm{HbA} 1 \mathrm{c}$ and blood glucose reduction in patients with type 2 diabetes.

\section{Disclosure}

The authors report no conflicts of interest in this work.

\section{References}

1. International Diabetes Federation. Available from: http://www.idf.org/ sites/default/files/Atlas-poster-2014_EN.pdf. Accessed September 25, 2015.

2. Inzucchi S, Buse J, Ferrannini E, et al. Management of hyperglycemia in type 2 diabetes, 2015: A patient-centered approach: update to a position statement of the American Diabetes Association and the European Association for the Study of Diabetes. Diabetes Care. 2015;38: 140-149.

3. Stark Casagrande S, Fradkin JE, Saydah SH, Rust KF, Cowie CC. The prevalence of meeting $\mathrm{A} 1 \mathrm{C}$, blood pressure, and LDL goals among people with diabetes, 1988-2010. Diabetes Care. 2013;36(8): 2271-2279.

4. National Institute for Health and Clinical Excellence. The Management of Type 2 Diabetes, NICE Clinical Guideline 87; 2014. Available from: https://www.nice.org.uk/guidance/cg87/resources/guidance-type-2diabetes-pdf. Accessed September 25, 2015.

5. Ali MK, Bullard KM, Saaddine JB, Cowie CC, Imperatore G, Gregg EW. Achievement of goals in U.S. diabetes care, 1999-2010. N Engl J Med. 2013;368:1613-1624.

6. Eng C, Kramer CK, Zinman B, Retnakaran R. Glucagon-like peptide-1 receptor agonist and basal insulin combination treatment for the management of type 2 diabetes: a systematic review and meta-analysis. Lancet. 2014;384(9961):2228-2234.

7. Diamant M, Nauck MA, Shaginian R, et al; 4B Study Group. Glucagonlike peptide 1 receptor agonist or bolus insulin with optimized basal insulin in type 2 diabetes. Diabetes Care. 2014;37:2763-2773.

8. Buse JB, Bergenstal RM, Glass LC, et al. Use of twice-daily exenatide in basal insulin-treated patients with type 2 diabetes: a randomized, controlled trial. Ann Intern Med. 2011;154:103-112.

9. Carris NW, Taylor JR, Gums JG. Combining a GLP-1 receptor agonist and basal insulin: study evidence and practical considerations. Drugs. 2014;74:2141-2152.

10. Grieg SL, Scott LJ. Insulin degludec/liraglutide: a review in type 2 diabetes. Drugs. 2015;75:1523-1534.

11. Sorli C. New developments in insulin therapy for type 2 diabetes. Am J Med. 2014;127(10 Suppl):S39-S48.

12. Vora J, Cariou B, Evans M, et al. Clinical use of insulin degludec. Diabetes Res Clin Pract. 2015;109(1):19-31.

13. Haahr H, Heise T. A review of the pharmacological properties of insulin degludec and their clinical relevance. Clin Pharmacokinet. 2014; 53:787-800.

14. Hollander P, King AB, Del Prato S, et al. Insulin degludec improves long-term glycaemic control similarly to insulin glargine but fewer hypoglycaemic episodes in patients with advanced type 2 diabetes on basalbolus insulin therapy. Diabetes Obes Metab. 2015;17(2):202-206.

15. Davies MJ, Gross JL, Ono Y, et al. Efficacy and safety of insulin degludec given as part of basal-bolus treatment with mealtime insulin aspart in type 1 diabetes: a 26-week randomized, open-label, treat-to-target non-inferiority trial. Diabetes Obes Metab. 2014;16(10):922-930.
16. Rodbard HW, Cariou B, Zinman B, et al. Comparison of insulin degludec with insulin glargine in insulin-naïve subjects with type 2 diabetes: a 2-year randomized, treat-to-target trial. Diabet Med. 2013; 30(11):1298-1304.

17. Eli Lilly and Company. Trulicity ${ }^{\mathrm{TM}}$ dulaglutide: US prescribing information 2016. Available from: http://pi.lilly.com/us/trulicity-uspi.pdf. Accessed February 20, 2016.

18. Fujishiro M, Izumida Y, Takemiya S, et al. A case of insulin allergy successfully managed using multiheximer-forming insulin degludec combined with liraglutide. Diabet Med. Epub 2015 Oct 20.

19. Scott LJ. Liraglutide: a review of its use in adult patients with type 2 diabetes mellitus. Drugs. 2014;74:2161-2174.

20. Bode B. An overview of the pharmacokinetics, efficacy, and safety of liraglutide. Diabetes Res Clin Pract. 2012;97:27-42.

21. Novo Nordisk A/S. Victoza liraglutide (rDNA origin) injection: US prescribing information. 2015. Available from: http://www.novo-pi. com/victoza.pdf. Accessed September 28, 2015.

22. Garber A, Henry R, Ratner R, et al. Liraglutide versus glimepiride monotherapy for type 2 diabetes (LEAD-3 Mono): a randomised, 52-week, phase III, double-blind, parallel-treatment trial. Lancet. 2009; 373(9662):473-481.

23. Novo Nordisk A/S. Xultophy ${ }^{\circledR}$ (NN9068). Available from: http:// www.novonordisk.com/rnd/pipelinedetails.1428300648863_2.html. Accessed February 21, 2016.

24. Electronic Medicine Compendium (eMC). Available from: https:// www.medicines.org.uk/emc/medicine/29493. Accessed February 21, 2016.

25. Kaptiza C, Bode B, Ingwersen SH, Jacobsen LV, Poulsen P. Preserved pharmacokinetic exposure and distinct glycemic effects of insulin degludec and liraglutide in IDegLira, a fixed-ratio combination therapy. J Clin Pharmacol. 2015;55(12):1369-1377.

26. Gough SC, Bode B, Woo V, et al; NN9068-3697 (DUAL-I) trial investigators. Efficacy and safety of a fixed-ratio combination of insulin degludec and liraglutide (IDegLira) compared with its components given alone: results of a phase 3, open-label, randomised, 26-week, treat-to-target trial in insulin-naive patients with type 2 diabetes. Lancet Diabetes Endocrinol. 2014;2:885-893.

27. Gough SC, Bode B, Woo VC, et al. One-year efficacy and safety of a fixed combination of insulin degludec and liraglutide in patients with type 2 diabetes: results of a 26-week extension to a 26-week main trial. Diabetes Obes Metab. 2015;17(10):965-973.

28. Buse JB, Vilsbøll T, Thurman J, et al; NN9068-3912 (DUAL-II) Trial Investigators. Contribution of liraglutide in the fixed-ratio combination of insulin degludec and liraglutide (IDegLira). Diabetes Care. 2014;37:2926-2933.

29. Mathieu C, Rodbard HW, Cariou B, et al; BEGIN: VICTOZA ADD-ON (NN1250-3948) study group. A comparison of adding liraglutide versus a single daily dose of insulin aspart to insulin degludec in subjects with type 2 diabetes (BEGIN: VICTOZA ADD-ON). Diabetes Obes Metab. 2014;16:636-644.

30. Freemantle N, Mamdani M, Vilsbøll T, Kongsø JH, Kvist K, Bain SC. IDegLira versus alternative intensification strategies in patients with type 2 diabetes inadequately controlled on basal insulin therapy. Diabetes Ther. 2015;6(4):573-591.

31. American Diabetes Association. Standards of medical care in diabetes 2016. Diabetes Care. 2016;39(Suppl 1):S1-S112.

32. Garber AJ, Abrahamson MJ, Barzilay JI, et al. Consensus statement by the American Association of Clinical Endocrinologists and American College of Endocrinology on the comprehensive type 2 diabetes management algorithm - 2016 executive summary. Endocr Pract. 2016;22: 84-113.

33. Freemantle N, Meneghini L, Christensen T, Wolden ML, Jendle J, Ratner R. Insulin degludec improves health-related quality of life (SF-36) compared with insulin glargine in people with type 2 diabetes starting on basal insulin: a meta-analysis of phase 3 a trials. Diabet Med. 2013;30:226-232. 
34. Freemantle N, Evans M, Christensen T, Wolden ML, Bjorner JB. A comparison of health-related quality of life (health utility) between insulin degludec and insulin glargine: a meta-analysis of phase 3 trials. Diabetes Obes Metab. 2013;15:564-571.

35. Home PD, Meneghini L, Wendisch U, et al. Improved health status with insulin degludec compared with insulin glargine in people with type 1 diabetes. Diabet Med. 2012;29(6):716-720.
36. Yavuz DG, Ozcan S, Deyneli O. Adherence to insulin treatment in insulin-naïve type 2 diabetic patients initiated on different insulin regiments. Patient Prefer Adherence. 2015;9:1225-1231.

37. Buysman EK, Liu F, Hammer M, Langer J. Impact of medication adherence and persistence on clinical and economic outcomes in patients with type 2 diabetes treated with liraglutide: a retrospective cohort study. Adv Ther. 2015;32:341-355.

\section{Publish your work in this journal}

Therapeutics and Clinical Risk Management is an international, peerreviewed journal of clinical therapeutics and risk management, focusing on concise rapid reporting of clinical studies in all therapeutic areas, outcomes, safety, and programs for the effective, safe, and sustained use of medicines. This journal is indexed on PubMed Central, CAS,
EMBase, Scopus and the Elsevier Bibliographic databases. The manuscript management system is completely online and includes a very quick and fair peer-review system, which is all easy to use. Visit http://www.dovepress.com/testimonials.php to read real quotes from published authors.

Submit your manuscript here: http://www.dovepress.com/therapeutics-and-clinical-risk-management-journal 